\title{
SINDROME DE KLIPPEL-TRENAUNAY-PARKES-WEBER COM ANGIOMATOSE MEDULAR
}

\author{
James Pitagoras de Mattos *
}

Até a presente data foi registrada na literatura a nosso alcance apenas um caso de síndrome de Klippel-Trénaunay-Parkes-Weber associada a angiomatose medular. A oportunidade que se nos apresentou de estudar exemplar mórbido com tal associação justifica a apresentação deste trabalho.

Numerosas referências bibliográficas a propósito de compressões medulares determinadas por angiomas são encontradas na literatura tais como as referidas por Guillain, Schmit \& Bertrand (1932) ${ }^{\&}$, Marinesco \& Draganesco (1935) ${ }^{13}$, Turner \& Kernohan (1941) ${ }^{18}$, Borges Fortes (1944) ${ }^{3}$, Epstein, Beller \& Cohen $(1948)^{6}$, porém, estes casos não se acompanhavam de manifestações cutâneas. O primeiro caso relatado de angiomatose cutâneo-medular é o de Cushing (1906) ${ }^{4}$, ocupando, no dorso, o dermátomo superposto a uma hemangioma pial.

A primeira referência bibliográfica sobre casos de hipertrofia corporal acompanhada de alterações vasculares foi feita por Trélat \& Monod (1869) ${ }^{1}$, que verificaram que frequentemente a hipertrofia se acompanhava de dilatações vasculares acometendo os capilares (nevus) e as veias subcutâneas (varizes); entretanto, estes autores não chegaram a individualizar tais alterações numa sindrome.

Ulteriormente, Klippel \& Trénaunay " descreveram, em 1900, sob o nome de nevus-ósteo-varicoso-hipertrófico, uma síndrome caracterizada pela seguinte triade sintomática: angioma subcutâneo plano, varizes congênitas e hipertrofia tissular. Observaram estes autores que os angiomas eram do tipo plano e que se localizavam, habitualmente, no segmento hipertrofiado. As varizcs eram inaparentes ao nascimento, sendo o seu desenvolvimento rápido e ocorrendo geralmente em torno da puberdade. A hipertrofia tissular pode ser segmentar ou total, manifestando-se sobretudo no sentido longitudinal. Além da forma completa da sindrome, descreveram três formas frustas (forma anévica, forma osteohipertrófica c forma avaricosa).

Em 1918, Parkes-Weber 11 descreveu a "hoemangiectatic hypertrophy" lembrando que as hipertrofias dos membros inferiores podem se acompanhar também de aneurismas artério-renosos ou cirsóides, ou de angiomas. Par-

Trabalho realizado no Instituto de Neurologia Deolindo Couto, Servico do Prof Bernardo Couto: $*$ Auxiliar de Ensino. 
kes-Weber considerava a sindrome de Klippel-Trénaunay, anteriormente descrita, como forma atenuada da grande familia das flebarterietasias hipertróficas, colocando o fator arterial em segundo plano.

Hallervorden ${ }^{y}$, em 1923, referiu-se a um caso de hipertrofia cerebral marcada na síndrome de Klippel-Trénaunay-Parkes-Weber.

A primeira venografia em casos de síndrome de Klippel-Trénaunay foi feita por Servelle ${ }^{16}$, em 1942. Ulteriormente, outras foram realizadas, observando-se bloqueio da circulação venosa com formação de varizes.

Poinso, Charpin \& Mme. Deprez ${ }^{15}$, em 1946, elaboraram duas teorias para explicar a hipertrofia (teoria vascular) e o angioma (teoria neuro-vegetativa). A teoria vascular, sustentada por Servelle, implicaria em anomalias na rede artério-venosa primitiva; a hipertensão venosa e o excesso de aporte sanguíneo levariam à hipertrofia. A teoria neuro-vegetativa primitiva, já sustentada por outros autores, admitia uma lesão simpática. No caso apresentado havia, no membro hipertrofiado: hiperpulsatilidade, aceleração da corrente circulatória e tensão venosa baixa, parecendo indicar incapacidade do sistema nervoso simpático em promover controle vasoconstritor.

A participação do sistema arterial foi defendida por Van Bogaert \& Kegels (1947) 19, ao publicarem um caso de nevus varicoso osteohipertrófico.

Louis-Bar \& Legros ${ }^{12}$, em 1948, relataram um caso de sindrome de Klippel-Trénaunay em criança de três anos e meio que, desde a idade de seis meses, apresentava crises de ausência. No mesmo trabalho relataram outro caso associado a neuroma plexiforme na face interna da coxa esquerda. Abundantes plexos terminais no interior dos nevus vasculares, foram observados, em 1949, por Weil, Bonnet \& Leveau ${ }^{21}$.

Em 1950, Aimes' diferenciou a sindrome de Klippel-Trénaunay da de Parkes-Weber, caracterizando a primeira pela triade sintomática constituída por nevus vascular plano, varicosidades e hipertrofia corporal e, a segunda, por apresentar, em lugar do nevus vascular plano, angiomas e aneurismas artério-venosos.

Lièvre ${ }^{11}$, em 1953, relacionou certos casos da sindrome de Klippel-Trénaunay com a doença de Von Recklinghausen. Além da associação acima citada, outras já foram descritas por diversos autores, assim como: sindactilia, aracnodactilia, polissindactilia, agenesia do $4 .^{\circ}$ rádio-metacarpodactilia, dedos em martelo, espina bífida, hipoplasia da primeira costela, luxação congênita da pelvis, uretra hipospádia, lipomatose, microcefalia e acrocefalia.

Quanto à etiologia da sindrome, cabe citar Becker (1969)\%, que se referiu a vários trabalhos com incidência familiar e a casos em parentes consanguíneos. Admite este autor, como fator principal um gen, talvez heredolábil, gens associados ou secundários e fatores peristásicos (endógenos e ambientais). 
Wiedmann, Tolksdorf, Hansen \& Klose (1969) ${ }^{22}$ estudaram os cromossomas de 8 doentes ( 2 homens e 6 mulheres) que exibiam a síndrome de Klippel-Trẻnaunay-Parkes-Weber, não observando qualquer alteração, excluindo, desta maneira, a hipótese de aberração cromossomial.

Djindjian, Hurt \& Houdart $(1973)^{5}$ relataram um caso semelhante ao nosso. Tratava-se de um paciente de 12 anos com a síndrome de Klippel-Trénaunay-Parkes-Weber, que apresentou hemorragia meníngea determinada por rotura de aneurisma artério-venoso medular em T10-T11. O caso descrito por estes autores e o exemplar mórbido que motivou a nossa publicação constituem, a nosso ver, os primeiros surgidos na literatura universal.

\section{OBSERVAÇAO}

D.A.C., 33 anos, sexo masculino, branco, solteiro, brasileiro, enfermeiro, internado em 16-02-71 (Reg. n. 21.856). Inicio da doença há 6 meses quando o pa('iente passou a sentir dormência no pé direito que ascendeu ao cabo de dias, até ao joelho. Trinta dias após, a mesma sensação apareceu no membro inferior esquerdo. Decorridos 2 meses, começou a ter dificuldade para andar, piorando lenta e progressivamente, chegando até a necessitar de apoio para a locomoção. Em seguida, experimentou periodo de melhora, voltando a andar sem apoio, continuando, porém, com a sensação de dormência. Atualmente encontra-se pior da marcha e começando a apresentar retenção urinária e fecal. Antecedentes pessoais e familiares - Nascido de parto normal e a termo. Sua mãe notou, logo após o nas(imento, manchas na pele de coloração vermelha que, com o passar dos anos, aumentaram de tamanho, chegando também a aparecer em outros territórios. Não há referência quanto à doença semelhante na família. Exame físico - A inspeção observamos manchas de coloração vermelha, irregulares, fazendo relevos, de tamanhos variáveis $(0,5 \mathrm{a} 2 \mathrm{~cm})$, únicas em algumas áreas, agrupadas em outras. atssim distribuídas: pequenas ao nivel da hemiface e pavilhão auricular $D$, o qual se apresenta alterado em sua forma, sendo maior que o $E$, e também na região costal D com transversal chegando a produzir abaulamento. Manchas semelhantes são também observadas em áreas dos membros superior e inferior $\mathrm{E}$, os quais se aßl esentam hipertrofiados (Fig. 1). Escoliose toráxica. Exame neurológico - Sinal de Romberg. Marcha pareto-espasmódica. Força diminuida nos membros inferioress. mormente a de flexão. Leve hipertonia elástica no membro superior direito Pacentuada nos membros inferiores. Os reflexos cutâneos-abdominais apresentam-se aholidos e os cutâneos-plantares em extensão (sinal de Babinski). Reflexos profundos vivos nos membros superiores e exaltados nos inferiores. Reflexo de Rossolimo presente bilateralmente. Sensibilidade: hipoestesia tátil, térmica e dolorosa deste T9-T10 para baixo; hipopalestesia desde as espinhas iliacas ântero-superiores jara baixo e hipobatiestesia nos membros inferiores (Fig. 2). Ao exame dos nervos (ranianos observamos, somente, reducão bitemporal do campo visual, mais acentuada it E. Troficidade -- hipertrofia do dimídio corporal $E$, encontrando-se as seguintes medidas:

\begin{tabular}{|c|c|c|c|c|}
\hline \multirow[t]{2}{*}{ segmentos } & \multicolumn{2}{|c|}{ Comprimento } & \multicolumn{2}{|c|}{ Perimetro } \\
\hline & direito & esquerdo & direito & esquerdo \\
\hline Brace & $26,5 \mathrm{~cm}$ & $28 \mathrm{~cm}$ & ('m & $1 \cdot \mathrm{m}$ \\
\hline Antebraco & $24 \quad \mathrm{~cm}$ & $24 \mathrm{~cm}$ & $25,5 \mathrm{c} \cdot \mathrm{m}$ & $\mathrm{c} \cdot \mathrm{m}$ \\
\hline Mão & 'm & $18 \mathrm{~cm}$ & $23 \quad \mathrm{~cm}$ & $\mathrm{c} \cdot \mathrm{m}$ \\
\hline Coxa & $\mathrm{cm}$ & $46 \cdot \mathrm{m}$ & $47 \quad \mathrm{~cm}$ & $48 \quad(\cdot \mathrm{m}$ \\
\hline Perna & $(\cdot \mathrm{m}$ & $3 S \cdot \mathrm{m}$ & $31.5 \mathrm{c} \cdot \mathrm{m}$ & $36,5 \quad \cdot \cdot \mathrm{m}$ \\
\hline Tornozelo & & & $21 \quad(\cdot \mathrm{m}$ & $23 \quad \mathrm{l} \cdot \mathrm{m}$ \\
\hline Pé & $23 . \overline{5} \mathrm{~cm}$ & $24 \mathrm{l} \cdot \mathrm{m}$ & & \\
\hline
\end{tabular}



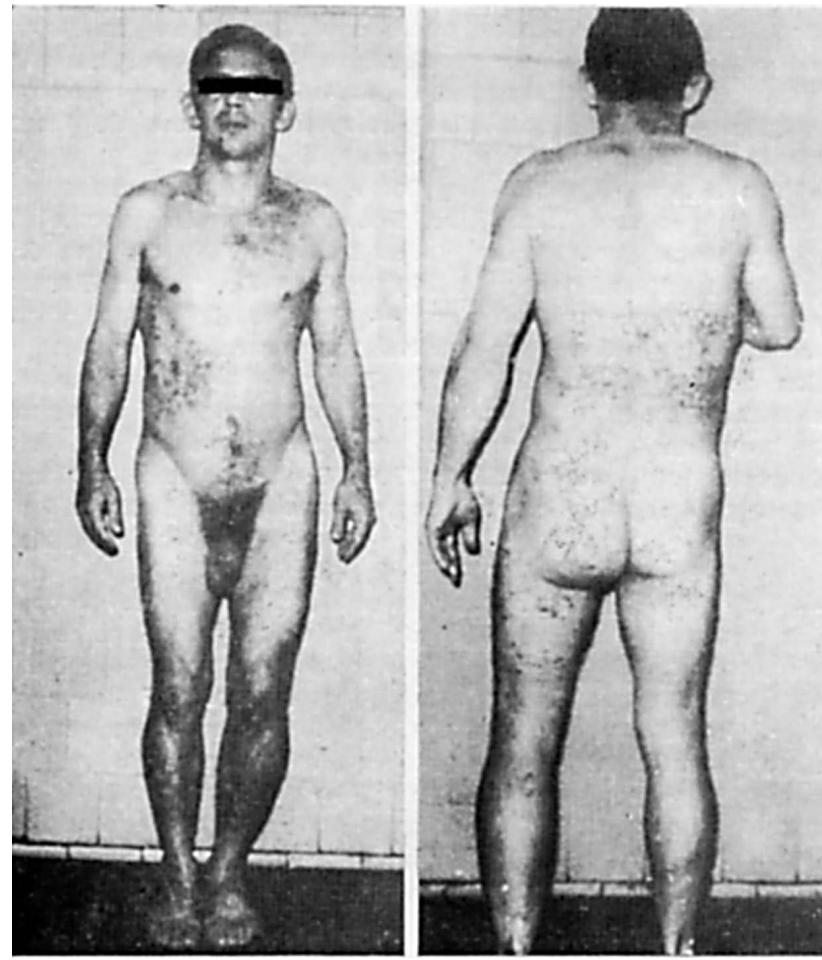

Fig. 1 - Caso D.A.C. Manchas de coloraçĩo vermelha, irregulares, fazendo relevos, unicas em algumas ireas, agrupadas em outras. Hipertrofia do membro inferior esquerdo.

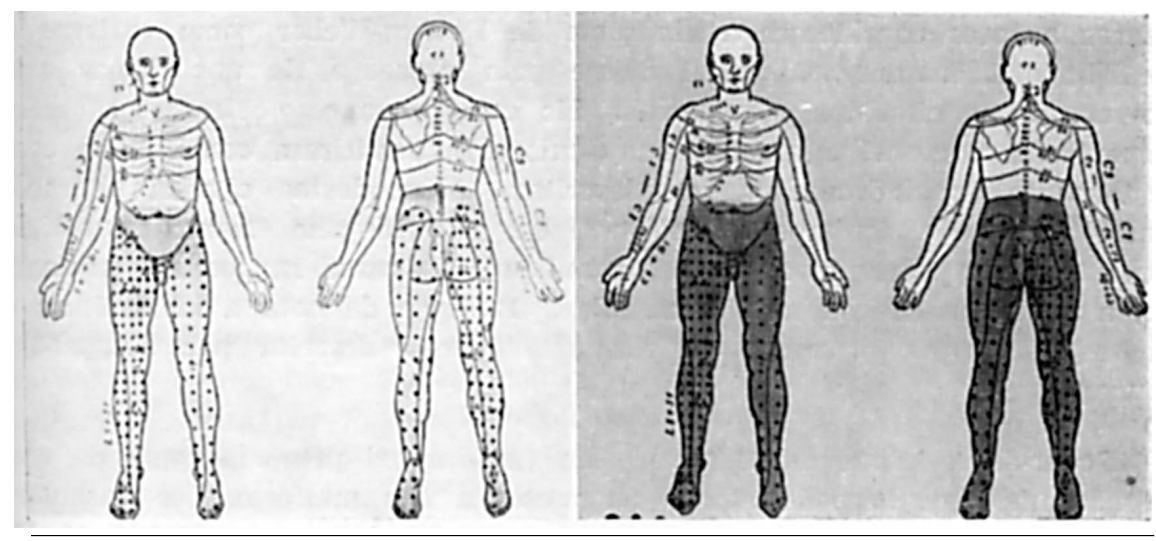

Fig. Z-Caso D.A.C. Hipoestesia tritil, térmica e delorosa do TG-T10 paia baixo. Hipopalestesia deste as espinhas iliaras antero-superiores para baixo. 
Exame do aparelho ćárdio-vascular - Pressão arterial nos 4 membros: membro superior esquerdo $120 / 90 \mathrm{~mm} \mathrm{Hg}$; membro inferior direito $130 \mathrm{~mm} \mathrm{Hg}$; membro inferior esquerdo $150 \mathrm{~mm} \mathrm{Hg}$. Não foì possivel o registro da pressão arterial mínima nos membros inferiores. Exames complementares - Os seguintes exames laboratoriais mostraram-se normais: hemograma, hemossedimentação, tempo de ('oagulação e sangramento, glicose, uréia, creatinina, colesterol, exame de fezes e de urina. Grupo sanguineo $\mathrm{O}$ fator $\mathrm{Rh}$ negativo. Exame do líquido cefalorraqueano: punçāo lombar em decúbito lateral; líquido límpido; a prova de Queekenstedt-Stookey mostrou bloqueio do espaço sub-aracnóide. Ecoencefalograma normal. Eletrencefalograma normal, com paroxismos lentos (ondas lentas de 6-7 c/s) na área fronto-temporal $\mathrm{E}$, acentuados e difundidos pela hiperpnéia (Dr. Ismar Fernandes). Eletrocardiograma normal. Exames radiológicos - Simples de crânio normal; da coluna tóraco-lombar mostrando lesōes degenerativas moderadas na coluna lombar e presença de nódulos de Schmol em T11, T12, L1 e L2; radiografias das articulações dos joelhos mostrando hipertrofia óssed à $\mathrm{E}$, constando de diferenças de $10 \mathrm{~mm}$ ao nivel do joelho e de $7 \mathrm{~mm}$ do nivel do terço superior da tibia. Mielografiu (Fig. 3) - Bloqueio total ao nivel de T9-T10; o limite superior da coluna de contraste apresenta-se irregular como nas imagens de angioma (Dr. Oswaldo Fernandes).

Evolucau - Como clinicamente tratava-se de sindrome de compressão medular, ronfirmada pelas provas de permeabilidade do canal raqueano e mielografia, indicamos a laminectomia. Entretanto, o paciente recusou-se à cirurgia, recebendo alta em 22-03-71. Ulteriormente, soubemos que o paciente havia sido submetido à laminectomia no Hospital de Ipanema (INPS). Entramos em contato com o Dr. Adherbal Maia que, gentilmente, se prontificou a nos ceder o resultado da angiografia medular e o laudo operatório. Em 15-0-72 foi realizada a angiografia medular, que mostrou angiomatose difusa (Fig. 3). Em 17-04-72, o paciente foi submetido à laminectomia (T7-T8-T9), evidenciando-se processo de aracnondite adesiva. A angiomatose difusa năo comprimia a medula.

\section{COMENTARIOS}

Sob a designação de nevus osteovaricoso-hipertrófico, Klippel \& Trénaunay descreveram a sindrome caracterizada pela triade: angioma subcutâneo plano, varizes congênitas e hipertrofia tissular. Além da forma completa, acrescentaram três formas frustas: anévica, osteohipertrófica e avaricosa. Outros autores consideram a síndrome de Parkes-Weber como variante da de Klippel-Trénaunay, da qual difere pela presença de aneurismas artério-venosos ou cirsóides e angiomas. No caso em apreço, elementos clínicos (hipertrofia corporal e anomalias vasculares) permitiram considerá-lo como pertencente às síndromes acima descritas. Além destas citadas alterações morfológicas e vasculares foi evidenciada, não só pelo exame neurológico como também pelas provas de permeabilidade do canal raqueano e mielografia, a sindrome de compressão medular. É digno de nota a inexistência de varizes, fato que o coloca na forma avaricosa descrita por Klippel-Trénaunay.

Poder-se-ia julgar o angioma como responsável pela sindrome de compressão medular, baseados tanto na presença das malformações vasculares e subcutâneas, como também nas imagens mielográfica e angiográfica. Entretanto, a exploração cirúrgica evidenciou que apesar da angiomatose ser difusa não determinava compressão da medula. Certamente, à conta da 

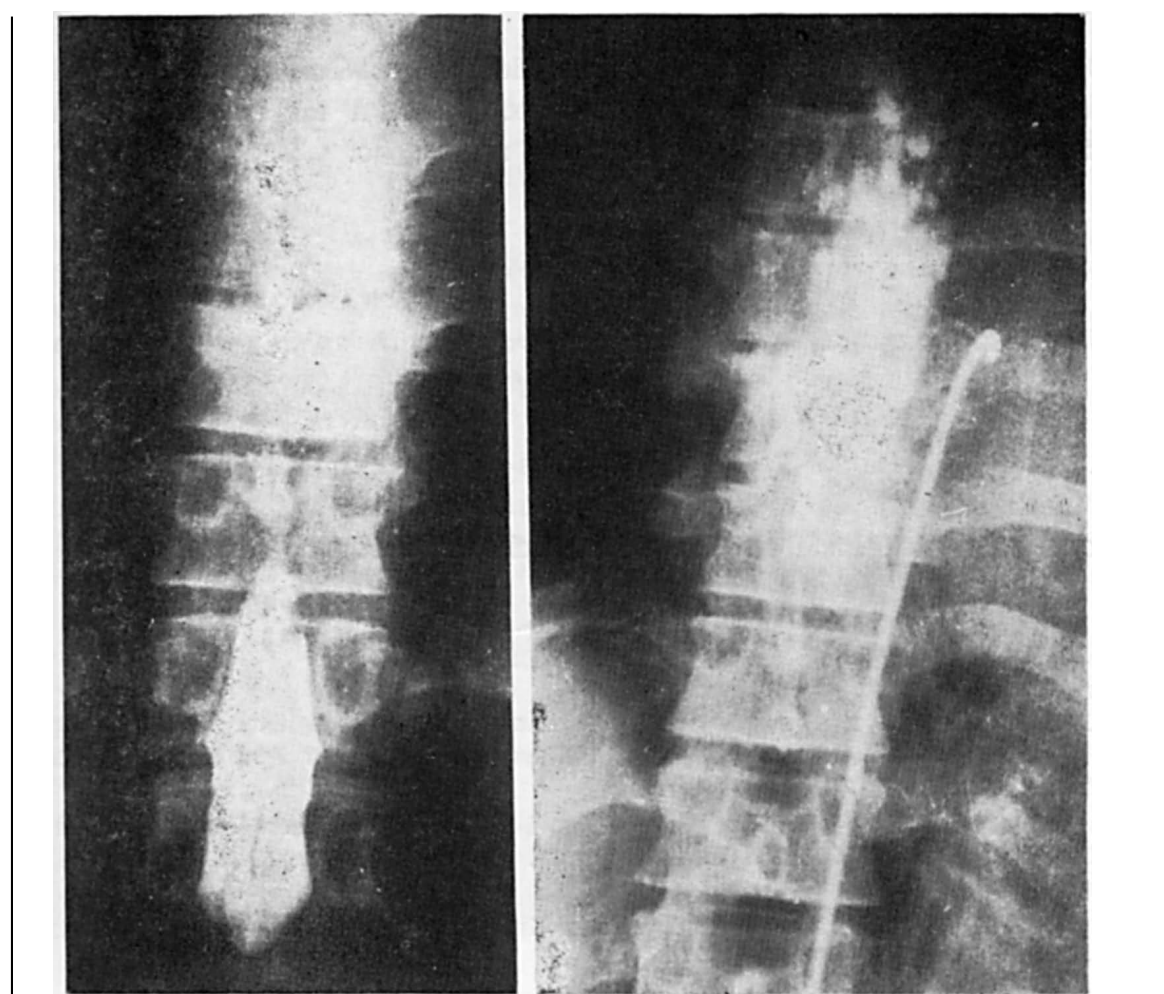

Fig. 3 -- Caso D.A.C. ì esquerda, mielografia mostrando bloqueio total do combraste ao nivel de T9-T10, sendo que o limite superior da coluna de contraste apresenta-se irregular, como nas imagens de angiomas; à direita, angiogratia medular mostrando angiomatose difusa.

aracnoidite adesiva secundária, encontrada no campo operatório, deve ser filiado o quadro compressivo medular. Sangramentos sucessivos anteriores devem ter determinado irritação e consecutivo espessamento da aracnóide espinhal.

A etiologia da sindrome de Klippel-Trénaunay-Parkes-Weber permanece obscura, já que foi excluída a hipótese de aberração cromossomial.

Alguns autores separam a sindrome de Klippel-Trénaunay da de Parkes-Weber, o que não coincide com a nossa opinião. Consideramos que a síndrome descrita por Parkes-Weber seja variante da de Klippel-Trénaunay, fato este anteriormente já descrito pelo primeiro autor.

Atualmente, há tendência em agrupá-las no grande capitulo das facomatoses, designando-as dentro da conceituação unitária, como síndrome de Klippel-Trénaunay-Parkes-Weber. 


\section{RESUMO}

E relatado um caso de sindrome de Klippel-Trénaunay-Parkes-Weber associada a angiomatose medular. O autor salienta ter encontrado na literatura somente uma referência com tal associação.

\section{SUMMARY}

Klippel-Trénaunay-Parkes-Weber syndrome with spinal cord angioma: a case report

A case of Klippel-Trénaunay-Parkes-Weber syndrome associated with spinal cord angioma is reported. The author points out that it was found only one reference in literature with such association.

\section{REFERENCIAS}

1. AIMES, A. - Syndrome de Klippel-Trénaunay. Hemangiectasie Hypertrophique de Parkes Weber. In Maladies et Syndromes Rares ou Peu Connues. 2e. ed., Masson et Cie., Paris, 1950.

2. BECKER, P. E. - Síndrome de Klippel-Trénaunay-Parkes-Weber. In Genética Humana. Tomo VII. Versão castelhana. Ediciones Toray S.A., Barcelona, 1969, págs. 86-91.

3. BORGES FORTES, E. - Angiomatose intra e extramedular. Cult. Med. (Rio de Janeiro) 6:253, 1944.

4. CUSHING, H. - Cit. por Djindjian \& col. $\because$

5. DJINDJIAN, R.; HURT, M. \& HOUDART, R. - Angiomes medullaires, dysplasies vasculaires ou géneralisées et phac'omatoses. Rev. neurol. (Paris) 124: 121. 1953.

6. EPSTEIN, J. A.; BELlER, A. J. \& COHEN, I. - Arterial anomalies on the spinal ('ord. J. Neurosurg. 6:45, 1949.

7. GUILl.AiN, G. \& ALAJOUANine, A. - Paraplégie par compression due a un volumineux angiocèle de la pie-mère spinale. J. Belge de Neurol, 25:689, 1925.

×. GLilllaiN, G.; SCHMIT, P. \& BERTRAND, I. - Hemangiome médulaire. Rerue neurol. (Paris) 57:420, 2932.

9. HAI,IERVORDEN, J. - Cit, por Becker :

111. KliPPEL, M. \& TRENAUNAY, P. - Cit. por Berker :

11. I.IEVRE. J. - Syndrome de Klippel-Trénaunay. Practique medico-chirulgic'ale i: $759,1953$.

12. LOLIS-BAR, D. \& LEGROS, J. - Les hypertrophies partielles aver: angiome isyndrome de Klippel-Trénaunay) et les rapports aver: la phacomatose. Conf. Neurol, $7: 245,1948$.

13. MARINESCO, G. \& DRAGANESCO, S. - Formations télangiectasiques méningées aver processus angiomateux intramedullaires. Rev. neurol. (Paris) 63: $8(19,1935$.

14. PARKES-WEBER, F. - Cit. por Berker :

15. POINSO, R.; CHARPIN. C. \& Mme. DEPREZ, D. - A propos d'un ras de noevus thoracobrachial avec ósteo-hypertrophie du membre superior droit isyndrome de Parkes-Weber). Prèsse Méd. 63:865. 1946. 
16. SERVELLE, M. - La veinographie va-t-elle nous permetre de demembrer le syndrome de Klippel-Trénaunay et l'hemangiectasie hypertrophique de Parkes-Weber? Prèsse Méd. 26:353, 1945.

17. TRÉLAT, U. \& MONOD, A. - Cit, por Becker

18. TURNER, O. R. \& KERNOHAN, J. W. - Vascular malformations and vascular tumors involving the spinal cord. Arch. Neurol. Psychiat. (Chicago) 46:444, 1941.

19. VAN BOGAERT, L. \& KEGELS, C. - Syndrome de Klippel-Trénaunay avec communications artério-veineuses. Travaux de l'Institut Bunge 5:93, 1947.

20. VIALE JR., S. M. - Venous angiomes of spinal cord. Arch. Neuro-Cir. Buenos Aires 2:46, 1945.

21. WEIL, G.; BONNET, G. \& LEVEAU, H. - Cit. por Becker :.

22. WIEIDMANN, H.; TOLSKSDORF, M.: HANSEN, H.\& KLOSE. K. - Cit. por Becker :.

Instituto de Neurologia Deolindo Couto - Av. Venceslau Braz 95, Botafogo -20000 Rio de Janeiro, GB - Brasil. 